Jeremy J. Ramsden

\title{
Nanotechnology and Gaia
}

ABSTRACT. The world's environmental problems are adumbrated. Strong attention has been directed towards nanotechnology, because it has been asserted that it will enable the environment to be repaired to a pristine state. Although the environment has traditionally been divided into the geosphere and the biosphere, comprising, respectively, the inanimate and the animate, the two are inseparably linked. This forms a central tenet of Gaia theory, which is critically examined. The present state of the environment is then examined in more detail. A brief survey of nanotechnology is given, followed by an assessment how it could be used to arrest environmental degradation. The final section examines what technology in general, of which nanotechnology is the latest and perhaps ultimate manifestation, can hope to achieve for humanity.

Nanotechnology Perceptions 10 (2014) 173-189 